\title{
A High Peculiarity Rate for Type Ia SNe
}

\author{
W. D. Li*, A. V. Filippenko*, A. G. Riess*1, \\ J. Y. $\mathrm{Hu}^{\dagger}$, and Y. L. $\mathrm{Qiu}^{\dagger}$ \\ ${ }^{*}$ Department of Astronomy, University of California, Berkeley, CA 94720-3411 USA \\ email: (wli, alex)@astro.berkeley.edu \\ ${ }^{\dagger}$ Beijing Astronomical Observatory, Chinese Academy of Sciences, Beijing 100080 China
}

\section{INTRODUCTION}

Type Ia supernovae (SNe Ia) are not perfectly homogeneous. There are peculiar ones: SN 1991T-like (overluminous), SN 1986G-like (subluminous), and SN 1991bglike (very subluminous) objects. Figure 1 shows a comparison of the spectra of peculiar SNe Ia with that of a relatively normal SN Ia, SN 1994D.

The peculiarity rate, however, is not well established. An estimate (less than 10\%) by Branch et al. (1993) [1] is limited by the small number of peculiar SNe Ia known at that time.

In the past 3 years, a number of peculiar SNe have been discovered in the course of several successful nearby SN surveys, and we try to update the peculiarity rate here.

\section{THE SN IA SAMPLE}

We have compiled a sample of 90 SNe Ia from 1997 to 1999 (up to SN 1999da). The only criterion we used to select SNe Ia in the sample is that the redshift of the SN must be smaller than 0.1. This is to avoid high-redshift SNe Ia to ensure that there is no contamination from possible evolution and/or observational biases between nearby and high-redshift SNe Ia.

The SNe Ia in our sample are subclassified as normal or as one of the peculiar SNe Ia: SN 1991T, which had prominent Fe III absorption lines and weak Si II lines prior to and near maximum brightness [2, 3]; SN 1991bg, which had an enhanced Si II $5700 \AA$ absorption, and a broad absorption trough extending from about 4150 to $4400 \AA$ due to Ti II lines [4, 5]; SN 1986G, which also had Ti absorption but less prominent than in SN 1991bg [6, 7]. Classification is done based on information in the International Astronomical Union Circulars (IAUC) and our SN spectrum and photometry database.

1) Now at Space Telescope Science Institute, Baltimore, MD 21218 


\section{THE OBSERVED PECULIARITY RATE}

We have divided our SN Ia sample into several subsamples and reported the observed peculiarity rates as follows.

In the total sample, all $90 \mathrm{SNe}$ are considered. 17 (18.9\%) SNe are peculiar, among which 11 (12.2\%) SNe are SN 1991T-like and 6 (6.7\%) SNe are SN $1991 \mathrm{bg} / 1986 \mathrm{G}$-like.

In the near-maximum sample, only the SNe Ia that were spectroscopically classified at no later than a week after maximum are considered. $61 \mathrm{SNe}$ are in the sample, among which $17(27.9 \%)$ are peculiar. 11 (18.0\%) SNe are SN 1991T-like and $6(9.9 \%)$ SNe are SN 1991bg/1986G-like.

In the Lick-Beijing (LB) sample, only the SNe that were discovered in the sample galaxies of the Lick Observatory Supernova Search (LOSS) and the Beijing Astronomical Observatory Supernova Survey (BAOSS) are considered. 35 SNe are in the sample, among which $13(37.1 \%)$ are peculiar. 7 (20.0\%) SNe are SN 1991T-like and $6(17.1 \%)$ SNe are SN 1991bg/1986G-like.

\section{OBSERVATIONAL BIASES}

There are various observational biases that make the observed peculiarity rate deviate from its intrinsic value.

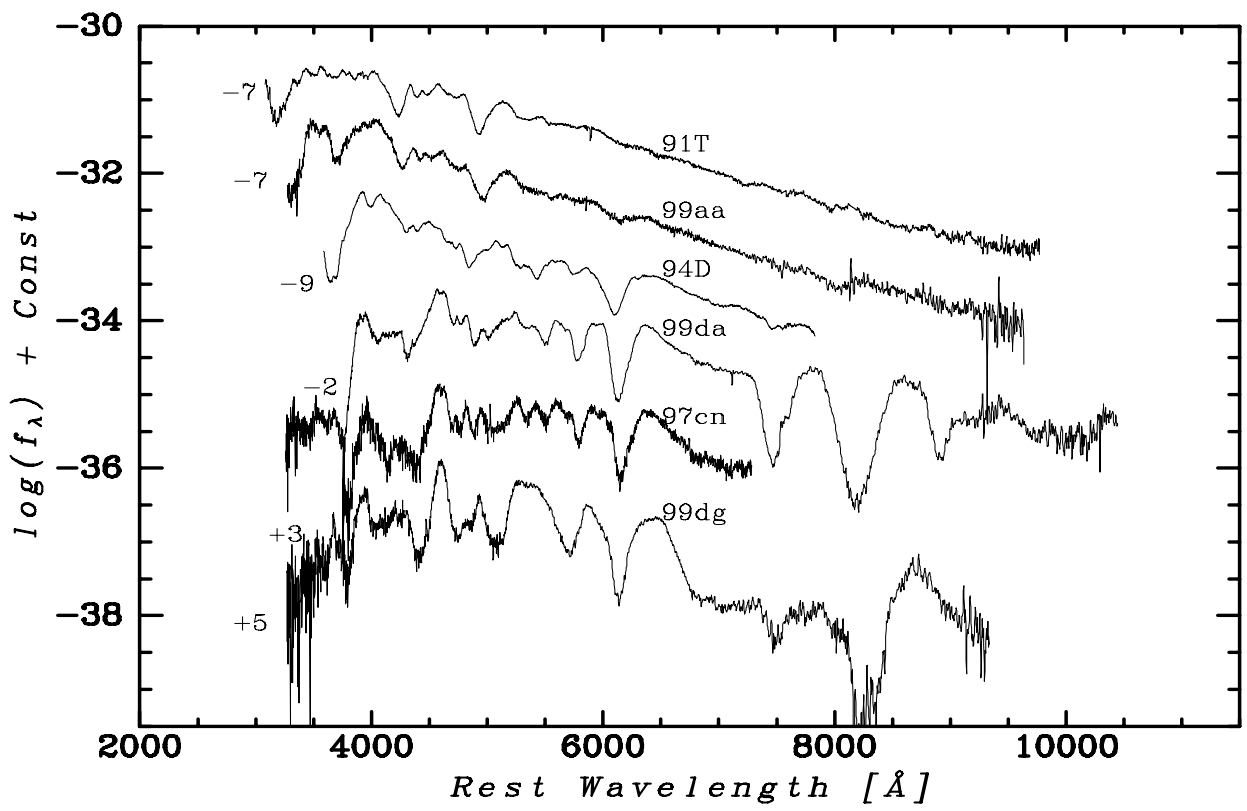

FIGURE 1. Spectra of two SN 1991T-like objects (99aa, 91T), one normal object (94D), and three SN 1991bg-like objects (99da, 97cn, 99dg). Days are relative to maximum. 
1. The maximum-only bias - caused by the fact that SN 1991T-like objects can best be spectroscopically identified prior to or near maximum brightness. It is thus unknown whether a SN discovered well after maximum is normal or SN 1991T-like. Classifying them as normal causes the maximum-only bias that underestimates the peculiarity rate.

2. The Malmquist bias - caused by the difference in luminosity among SNe Ia.

3. The light-curve shape bias - caused by the difference in light-curve shape among SNe Ia.

\section{MONTE CARLO SIMULATIONS}

We have done Monte Carlo simulations to study the effects of the observational biases. Simulations are done for magnitude-limited SN surveys, with the baseline as the only input parameter. Simulations are also done for distance-limited SN surveys, with the baseline and the limiting magnitude of the survey as parameters.

All observational biases are well accounted for in the Monte Carlo simulations. We also studied the role of extinction of SN 1991T-like objects in determining the rate of those objects. There are speculations that SN 1991T-like objects are more

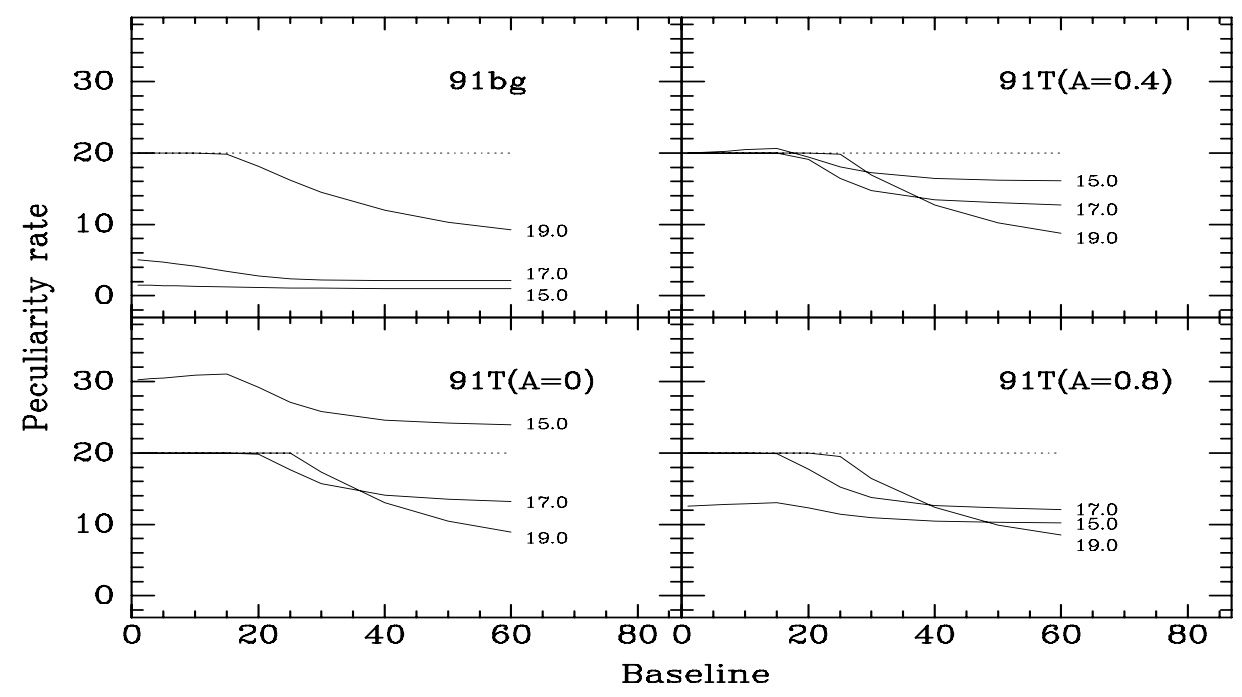

FIGURE 2. The peculiarity rate found in the Monte Carlo simulations for distance-limited SN surveys. Results are shown for different baselines and different limiting magnitudes, and for the case of SN 1991bg-like objects (upper left), SN 1991T-like objects with no extra extinction (lower left), SN 1991T-like objects with 0.4 mag of extra extinction (upper right), and SN 1991T-like objects with $0.8 \mathrm{mag}$ of extra extinction (lower right). The dotted line is the input (intrinsic) rate. 
likely to occur in dusty, star-forming regions and thus may suffer more extinction than the normal and SN 1991bg/1986G-like objects.

An example of the results of the Monte Carlo simulations is shown in Figure 2.

\section{THE INTRINSIC PECULIARITY RATE}

Our simulations indicate that all SNe Ia in the LB sample should have been discovered because the two surveys are distance-limited with small baselines and deep limiting magnitudes. In other words, the peculiarity rate and luminosity function in the LB sample should be intrinsic.

Our simulations also indicate that a high peculiarity rate (more than 30\%) and a flat luminosity function for SN Ia (e.g., the rate of SN 1991bg-like and SN 1991Tlike objects are comparable) are consistent with the observed peculiarity rates in all three (total, near-maximum, and LB) samples.

These results have important implications for studies of high-redshift SNe Ia and of SN Ia progenitor systems.

1. The high-redshift results: The high peculiarity rate for nearby SNe Ia $(>30 \%)$ and the small apparent (but preliminary) peculiarity rate for the several dozen high-redshift SNe Ia studied thus far [8, 9] may indicate a systematic difference between them. To reconcile the absence of SN 1991T-like objects found at high redshifts with the peculiarity rate found at low redshifts, an extra extinction of more than $\sim 1$ mag for the SN 199T-like objects is needed, which is not supported by the observations. However, the existing spectral studies of highredshift SNe Ia are not very detailed.

2. The progenitor systems of SNe Ia: The high peculiarity rate, together with other evidence, favors the existence of multiple progenitor systems for SNe Ia (e.g., single-degenerate systems and double-degenerate systems).

Our supernova research at UC Berkeley is supported by NSF grant AST-9417213 and NASA grant GO-7434.

\section{REFERENCES}

1. Branch, D., Fisher, A., and Nugent, P., A.J. 106, 2383 (1993).

2. Filippenko, A. V., et al., Ap.J. 384, L15 (1992a).

3. Phillips, M. M., et al., A.J. 103, 1632 (1992).

4. Filippenko, A. V., et al., A.J. 104, 1543 (1992b).

5. Leibundgut, B., et al., A.J. 105, 301 (1993).

6. Phillips, M. M., et al., P.A.S.P. 99, 592 (1987).

7. Cristiani, S., et al., A\&A 259, 63 (1992).

8. Riess, A. G., et al., A.J. 116, 1009 (1998).

9. Perlmutter, S., et al., Ap.J. 517, 565 (1999). 